\title{
Author Correction: From nanoscale interface characterization to sustainable energy storage using all-solid-state batteries
}

Darren H. S. Tan, Abhik Banerjee, Zheng Chen (1) and Ying Shirley Meng (1)

Correction to: Nature Nanotechnology https://doi.org/10.1038/s41565-020-0657-x, published online 10 March 2020.

In the version of this Review Article originally published, ref. ${ }^{68}$ (Zeng, X., Li, J. \& Ren, Y.) should not have been included; it has now been deleted from the reference list and its citation removed from the text. Subsequent references have been renumbered. Furthermore, in the final sentence in Fig. 3 caption ref. ${ }^{19}$ should have been the last in the list of references, as it relates to panel $\mathbf{f}$. The online versions of the Review Article have been amended.

Published online: 5 March 2021

https://doi.org/10.1038/s41565-021-00877-5

(C) Springer Nature Limited 2021 